\title{
Remanence measurements on individual magnetotactic bacteria using a pulsed magnetic field
}

\author{
Ietje Penninga a ${ }^{a}$ Hendrik de Waard a , Bruce M. Moskowitz ${ }^{b}$, \\ Dennis A. Bazylinski ${ }^{c}$, Richard B. Frankel ${ }^{d}$ \\ ${ }^{a}$ Department of Physics, University of Groningen, Nyenborgh 4, 9747 AG Groningen, The Netherlands \\ ${ }^{b}$ Institute for Rock Magnetism and Department of Geology and Geophysics, University of Minnesota, Minneapolis, MN 55455, USA \\ ${ }^{c}$ Marine Science Center, Northeastern University, East Point, Nahant, MA 01908, USA \\ ${ }^{d}$ Department of Physics, California Polytechnic State University, San Luis Obispo, CA 93407, USA
}

\begin{abstract}
We describe pulsed-magnetic-field remanence measurements of individual, killed, undisrupted cells of three different types of magnetotactic bacteria. The measurement technique involved the observation of aligned, individual magnetotactic bacteria with a light microscope as they were subjected to magnetic pulses of increasing amplitude. We show that for MM cells, the hysteresis loop is square, with the coercive field variable from cell to cell. This is consistent with just two magnetization states for the single chain of magnetite particles. For MR and MMP cells, the hysteresis loops are not square, indicating that there are several different magnetization states, and that individual cells can be demagnetized. The coercive fields in the MR and MMP cells are less variable than for the MM cells.
\end{abstract}

\section{Introduction}

Magnetotactic bacteria, a diverse group of prokaryotes, contain magnetosomes [1,2], which are magnetic mineral particles enclosed in membranes. In most cases, the magnetosomes are arranged in a single or multiple chains, and are apparently fixed within the cell. In many magnetotactic bacterial types, the magnetosome mineral particles consist of ferrimagnetic magnetite, $\mathrm{Fe}_{3} \mathrm{O}_{4}$ [3], are characterized by narrow particle size distributions, and have uniform, species-specific, crystalline habits [2]. The particle sizes range between 40 to $100 \mathrm{~nm}$, and are within the permanent single-magnetic-domain size range for magnetite [4].
In many magnetotactic bacteria from marine, sulfidic environments, the magnetosome particles consist of the iron-sulfide mineral greigite, $\mathrm{Fe}_{3} \mathrm{~S}_{4}$ [5], which is isostructural with magnetite and is also ferrimagnetically ordered at ambient temperature. The greigite particles are also characterized by narrow particle size distributions and species-specific crystal habits [6]. However, whereas the magnetite particles in a magnetosome chain are usually oriented so that a [111] crystallographic axis of each particle lies along the chain direction, the greigite particles in a magnetosome chain are usually oriented so that a [100] crystallographic axis of each particle is oriented along the chain direction. In one 
organism, an unusual, many-celled, magnetotactic prokaryote (MMP) [7], particles of non-magnetic pyrite, $\mathrm{FeS}_{2}$, occur in addition to $\mathrm{Fe}_{3} \mathrm{~S}_{4}$ [5]. In another organism, both magnetite and greigite particles occur in the same chain of magnetosomes [8].

Whether the mineral particles are magnetite or greigite, the chain of magnetosome particles constitutes a permanent magnetic dipole fixed within the bacterium [9]. The remanent moment is generally close to its saturation value. Normally it is sufficiently larger than the background thermal energy so that it, and consequently the bacterium, is oriented along geomagnetic field lines as it swims, causing the bacterium to migrate along the field lines.

Kalmijn and Blakemore [10] and Mizota and Maeda [11] have reported on the effect of pulsed magnetic fields on the migration of uncharacterized magnetotactic bacteria collected from natural environments. An intense magnetic pulse of several hundred gauss, oriented opposite to a steady, several gauss magnetic field in which the bacteria were migrating, caused a fraction of the bacteria, which increased over a certain range with the strength of the pulses, to reverse their direction of migration. Similarly, exposure of magnetotactic bacteria to an intense $60 \mathrm{~Hz}$ magnetic field caused about 50 percent of magnetotactic bacteria in a sample to migrate opposite to the original migration direction, subsequent to removal of the $60 \mathrm{~Hz}$ field [12].

These observations were consistent with only two, opposite, directions of magnetization relative to the chain direction and a square hysteresis loop for each cell, but with coercive fields that varied from cell to cell, or varied according to the instantaneous orientation of the cell with respect to the direction of the magnetic pulse.

In this paper, we describe pulsed-magnetic-field remanence measurements of individual, killed, undisrupted cells of three different types of magnetotactic bacteria. The measurement technique was based on a method described by Knowles [13] for small synthetic magnetic particles. It involved the observation of aligned, individual magnetotactic bacteria with a light microscope as they were subjected to magnetic pulses of increasing amplitude. The microorganisms studied were the culturable magnetotactic bacterium Magnetospirillum magnetotacticum (MM) (formerly Aquaspirillum magnetotacticum ATCC 31632), which contains a single chain of cubo-octahedral magnetite-containing magnetosomes [3], and two uncultured magnetotactic bacteria; an uncharacterized magnetotactic curved rod that contains two or more adjacent chains of greigite-containing magnetosomes (MR), and the MMP described above. The MR and MMP were collected from water and sediment obtained from a brackish salt marsh pool at Morro Bay, California.

As we show below, the MM hysteresis loop is square, with the coercive field variable from cell to cell. This is consistent with just two magnetization states for the single chain of magnetite particles. For the MR and MMP, the hysteresis loops are not

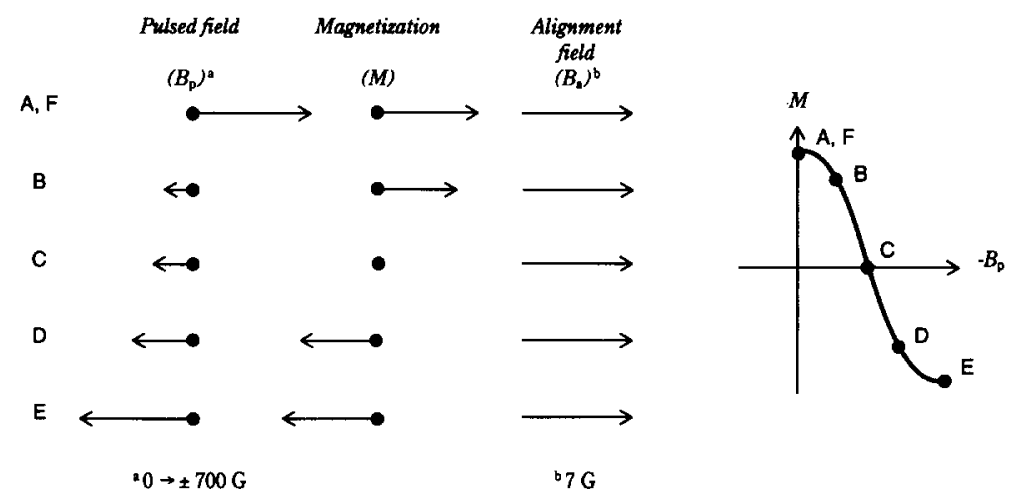

Fig. 1. Schematic diagram of the pulsed magnetic field remanence measurements. A cell aligned in the field $B_{\mathrm{a}}$ is subjected to high field pulses $B_{\mathrm{p}}$, mostly oriented opposite to $\boldsymbol{B}_{\mathrm{a}}$. Following each pulse, the relative remanent moment of the cell $\boldsymbol{M}_{\mathrm{r}}$ is determined as described in the text. A-F correspond to a sequence of pulses and remanent moment measurements that results in the remanence plot shown to the right. A, $B_{\mathrm{p}}=0 \mathrm{G} ; \mathrm{B}-\mathrm{E} ;-700 \mathrm{G}<B_{\mathrm{p}}<0 \mathrm{G} ; \mathrm{F}, B_{\mathrm{p}}=+700 \mathrm{G}\left(\boldsymbol{B}_{\mathrm{p}}\right.$ parallel to $\left.B_{\mathrm{a}}\right)$. 
square, indicating that there are several different magnetization states, and that individual cells can be demagnetized. The coercive fields in the MR and MMP cells are less variable than for the MM cells.

\section{Method}

Cells of MM were cultured as previously described [14] and fixed in 1\% glutaraldehyde. Some of the fixed cells were deposited on carbon-coated electron microscope grids for transmission electron microscopy.

Cells of MR and MMP were collected from brackish salt marsh sediments using a modified race-track technique [15]. The cells were fixed in 3\% formaldehyde. Some of the MR cells were deposited on carbon-coated electron microscope grids for transmission electron microscopy.

Magnetic measurements are shown schematically in Fig. 1. They were made with an Olympus BH-2 microscope fitted with a video camera, VCR and monitor, and a coil system. The $\times 40$ objective was modified by removing a magnetic spring that pressed on the lens and caused a distortion of the field at the object site. The metal microscope stage was replaced by a lucite stage that was fitted with a coil system consisting of insulated copper wire wound on lucite forms.

The coil system consisted of two orthogonal, 500-turn coil pairs (nominally oriented $\mathrm{N}-\mathrm{S}$ and $\mathrm{E}-\mathrm{W}$ ) that generated a variable (up to $15 \mathrm{G}$ ), DC alignment field $\boldsymbol{B}_{\mathrm{a}}$ in the optical plane of the microscope. The orientation of $\boldsymbol{B}_{\mathrm{a}}$ in the plane could be controlled by adjusting the currents through the two coil pairs. A field $\boldsymbol{B}_{\mathrm{a}}$ with constant magnitude could also be used, rotating in the plane at a variable rate by applying sinusoidal currents, 90 degrees out of phase, to the two coil pairs. Another 600-turn coil pair, oriented parallel to the E-W alignment pair, was used to generate a 1 to $5 \mathrm{~ms}$ magnetic pulse field $B_{\mathrm{p}}$ with variable amplitude up to $700 \mathrm{G}$. The two pulse coils, calibrated for field as a function of current, were connected through switching transistors to a 50-150 V power supply. The magnitude of the pulse field was obtained from the reading of a peak voltmeter that measured the sum of the voltages across standard resistors in series with the coils.
Fixed cells in aqueous suspension were drawn up into a glass capillary tube with a rectangular crosssection and an optical path length of $0.1 \mathrm{~mm}$ (InVitro Dynamics). The capillary tube was placed on the stage and viewed through the microscope. The suspended cells were observed to orient along $\boldsymbol{B}_{\mathrm{a}}$, and to rotate by 180 degrees if the direction of $B_{a}$ was reversed by reversing the direction of current in the coils.

Most of the measurements were made with only the $\mathrm{E}-\mathrm{W}$ alignment coils activated, with $\mathrm{B}_{\mathrm{a}}$ approximately 7 G. Pulsed magnetic fields of increasing amplitude with $\boldsymbol{B}_{\mathrm{p}}$ oriented opposite to $\boldsymbol{B}_{\mathrm{a}}$ were applied to the sample. When $B_{p}$ exceeded the coercive force $H_{\mathrm{c}}$ of the chain of particles in a particular cell, the cell could be seen to rotate by 180 degrees following the pulse.

The remanent magnetic dipole moment of the cell $\mathrm{M}_{\mathrm{r}}$, following a pulse of amplitude $B_{\mathrm{p}}$, relative to its original value, i.e., before being subjected to any pulses, was determined by measuring the time $T$ required for the cell to rotate between two angles, $\phi_{1}$ and $\phi_{2}$, with respect to $\boldsymbol{B}_{\mathrm{a}}$, following a reversal of $\boldsymbol{B}_{\mathrm{a}}$. The relationship between the torque exerted by $\boldsymbol{B}_{\mathrm{a}}$ on $M_{\mathrm{r}}$ and the viscous drag on the cell as it rotates is given by [16]:

$M_{\mathrm{r}} B_{\mathrm{a}} \sin \theta=V_{\mathrm{d}} \mathrm{d} \theta / \mathrm{d} t$,

where $\theta$ is the instantaneous angle between $M_{\mathrm{r}}$ and $B_{\mathrm{a}}$, and $V_{\mathrm{d}}$ is the rotational-viscous-drag coefficient, which for a sphere is equal to $8 \pi \eta R^{3}$ ( $\eta$ is the viscosity coefficient of water and $R$ is the radius of the sphere) [17]. Rearrangement and integration of Eq. (1) yields

$T=\left(V_{\mathrm{d}} / M_{\mathrm{r}} B_{\mathrm{a}}\right)\left[\ln \tan \left(\phi_{1} / 2\right)-\ln \tan \left(\phi_{2} / 2\right)\right]$.

For constant $V_{\mathrm{d}}, B_{\mathrm{a}}, \phi_{1}$ and $\phi_{2}, T$ is inversely proportional to $M_{\mathrm{r}}$. Because of the logarithmic divergence of $T$ at $\phi=0$ and $180^{\circ}, T$ was measured between $\phi_{1}=10^{\circ}$ and $\phi_{2}=170^{\circ}$. This method could not be used to determine the absolute magnitude of the cellular remanent moment because the actual viscous drag coefficients for the cells in the water medium were not known.

A variation on this method utilized $B_{\mathrm{a}}$ rotating in the focal plane at a fixed frequency $(\approx 0.5 \mathrm{~Hz})$ [18], initially with a field magnitude of approximately 7 


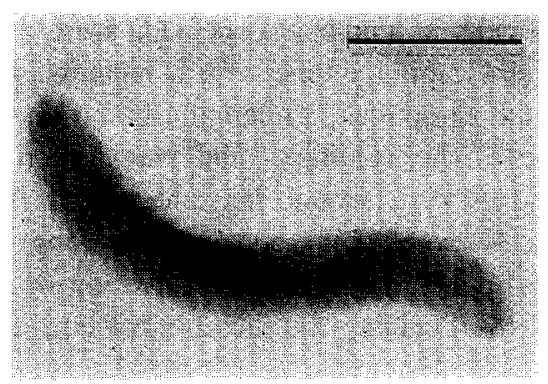

Fig. 2. Transmission electron micrograph of a Magnetospirillum magnetotacticum $(\mathrm{MM})$ cell. $\mathrm{Bar}=1 \mu \mathrm{m}$.

G. At first the cell rotated at constant angular velocity with the field. Then, with the rotation frequency unchanged, the field magnitude $B_{\text {a }}$ was slowly reduced until at a value $\left(B_{\mathrm{a}}\right)_{\min }$, the cell was observed to stop rotating with the field. According to Eq. (1), rotation of a cell at constant angular velocity will occur as long as the torque on the cell due to the rotating field is balanced by the viscous drag. As $B_{\mathrm{a}}$ is decreased, $\theta$ increases. At $B_{\mathrm{a}}=\left(B_{\mathrm{a}}\right)_{\min }, \theta=90^{\circ}$; for $B_{\mathrm{a}}<\left(B_{\mathrm{a}}\right)_{\min }$, the cell stops rotating with the field. From Eq. 1, $M_{\mathrm{r}}$ is proportional to $\left(B_{\mathrm{a}}\right)_{\min }$. As noted above, since $V_{\mathrm{d}}$ was not known, only the relative magnitude of the remanent moment $M_{\mathrm{r}}$ of the cell could be determined in this way.

Besides the pulse measurements described above, the average coercive force and remanence curves of a bulk sample of the cultured MM cells were measured with an alternating gradient force magnetometer in static fields up to $1.0 \mathrm{kG}$. The isothermal remanent magnetization (IRM) curve was deter-

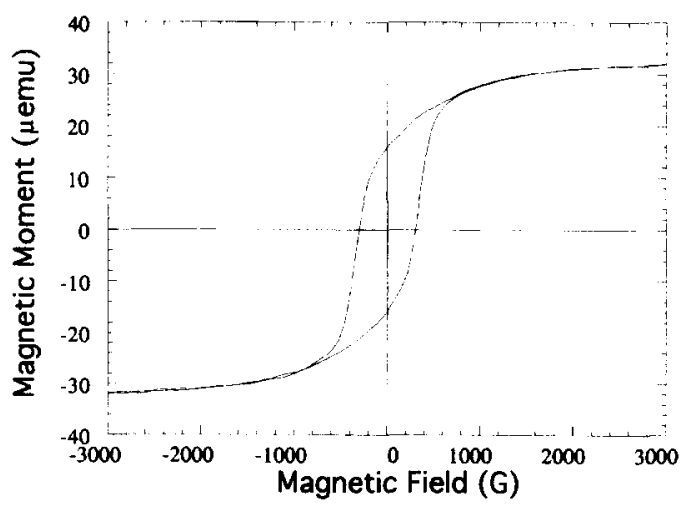

Fig. 3. Hysteresis loop for a bulk MM sample at $295 \mathrm{~K}$. The coercive force is $303 \mathrm{G}$. mined by applying progressively larger dc fields to an initially demagnetized sample and measuring the remanence after removal of the field. The dc demagnetization (DCM) curve was determined by saturating the sample in a large positive field and measuring the remanence after applying progressively larger, negative, dc fields. The bulk sample was prepared by drying a small drop of the cell suspension onto a glass cover slip.

\section{Results}

\subsection{Magnetospirillum magnetotacticum}

An electron micrograph of one of the MM spirilla with its single chain of cubo-octahedral magnetite particles is shown in Fig. 2. Examination of thirty micrographs showed that the cells contained from 8 to 52 magnetosomes (ave. 24) with an average particle volume of $9.6 \times 10^{-17} \mathrm{~cm}^{3}$. Using the value of $480 \mathrm{emu} / \mathrm{cm}^{3}$ for $\mathrm{Fe}_{3} \mathrm{O}_{4}$ at $295 \mathrm{~K}$, this yields an estimated magnetic moment per cell ranging from $M=0.32$ to $2.4 \times 10^{-12} \mathrm{emu}$, with an average value of $1.1 \times 10^{-12} \mathrm{emu}$ ( $1 \mathrm{emu}=1 \mathrm{erg} /$ gauss). The bulk coercive force at $295 \mathrm{~K}$ was $303 \mathrm{G}$ (Fig. 3), which is consistent with previous measurements on MM cells [19]. The remanence ratio $\left(M_{\mathrm{r}} / M_{\mathrm{s}}\right)$ was 0.48 , in excellent agreement with the theoretical value of 0.5 for a random dispersion of single-magnetic-domain particles.

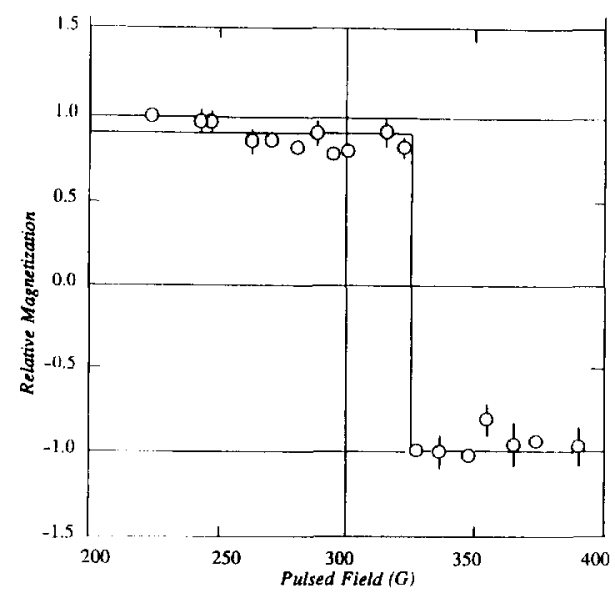

Fig. 4. Pulsed magnetic field remanence plot for an individual MM cell. 
In the pulsed remanence curve of Fig. 4, the relative magnitude of the remanent dipole moment of a cell $M_{\mathrm{r}}$ is plotted against the amplitude $B_{\mathrm{p}}$ of the pulsed magnetic field. Since $\boldsymbol{B}_{\mathrm{a}}$ was taken as defining the positive direction, and $\boldsymbol{B}_{\mathrm{p}}$ is antiparallel to $\boldsymbol{B}_{\mathrm{a}},-\boldsymbol{B}_{\mathrm{p}}$ is plotted along the abscissa in Fig. 4. For negative values of $M_{\mathrm{r}}$ the magnetization of the chain has reversed direction within the cell. The hysteresis loop in this spirillum is very square. In such cases, the coercive field, $H_{c}$, is defined as the field at which the magnetization reversal takes place; for the present sample, $H_{\mathrm{c}}=310(3) \mathrm{G}$. A square hysteresis loop is characteristic of all MM cells, suggesting that the magnetization of a cell can be reversed, but that the cell cannot be demagnetized. In a few cells, however, the magnetization reversal occurred in two stages, with a sudden small decrease in moment followed by complete magnetization reversal at a slightly higher pulse field.

The magnitude of $M_{\mathrm{r}}$ for a cell was the same before and after a saturation pulse $\left(B_{\mathrm{p}}=700 \mathrm{G}\right)$, with $\boldsymbol{B}_{\mathrm{p}}$ parallel to $\boldsymbol{B}_{\mathrm{a}}$, indicating that the micromagnetic spin structure of the chain was the same in both states, and that the natural remanent moment of the cells equals the saturation remanent moment.

The IRM and DCM curves for the bulk sample are shown in Fig. 5. As a measure of inter-strand or intra-strand dipolar interactions, the two sets of remanence measurements are combined to form a

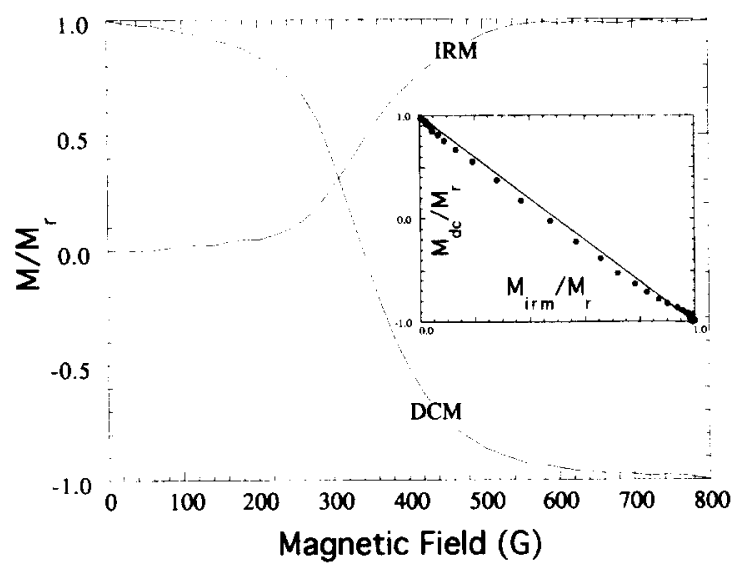

Fig. 5. Normalized curves of isothermal remanent magnetization (IRM) and dc demagnetization (DCM) for bulk sample. Curves have been normalized to the saturation remanence produced in 1 kG. A Henkel plot for this sample is shown in the insert.

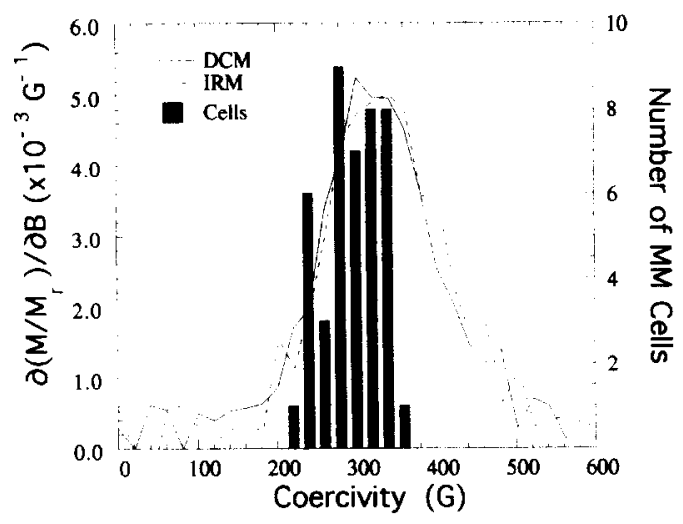

Fig. 6. Histogram of switching fields for MM cells with switching field distributions obtained by differentiating the DCM and IRM data in Fig. 5. The remanent SFDs have been normalized by the total area under each curve.

Henkel plot (e.g., Ref. [20]) and shown in the inset of Fig. 5. For non-interacting single domain particles, the Henkel plot is linear with a slope of -2 . The slight concave upward departure from ideal behavior suggests a minimal degree of negative (demagnetizing) interactions between magnetosome chains [19-22].

The pulse fields at which magnetization reversal occurred were measured for 50 spirilla and are displayed in the histogram shown in Fig. 6 . The average magnetization reversal field for these cells was 292 $\mathrm{G}$, in good agreement with the bulk coercivity of 303 $\mathrm{G}$ for the sample but less than the remanent coercivity $\left(H_{\mathrm{r}}\right)$ of $339 \mathrm{G}$. The solid and dashed lines in Fig. 6 were obtained by differentiating the DCM and IRM remanence plots of the bulk data with respect to magnetic field and provide estimates of the switching field distributions (SFD) during magnetization and remagnetization. Both remanent SFDs are approximately the same suggesting minimal interaction effects and is another expression of the near linear Henkel plot. Although the average coercivity is approximately the same for both the bulk and single cell experiments, there is a sharp cutoff in the single cell SFD compared to the high-field tail for the random dispersion of dried cells.

The dependence of $\mathrm{H}_{\mathrm{c}}$ on the orientation of $\boldsymbol{B}_{\mathrm{p}}$ with respect to the orientation of $\boldsymbol{M}_{\mathrm{r}}$ was investigated by comparing $H_{\mathrm{c}}$ for $\boldsymbol{B}_{\mathrm{p}}$ oriented antiparallel to $\boldsymbol{B}_{\mathrm{a}}$, to $H_{\mathrm{c}}$ for $\boldsymbol{B}_{\mathrm{p}}$ oriented at 45 degrees to $\boldsymbol{B}_{\mathrm{a}}$. 


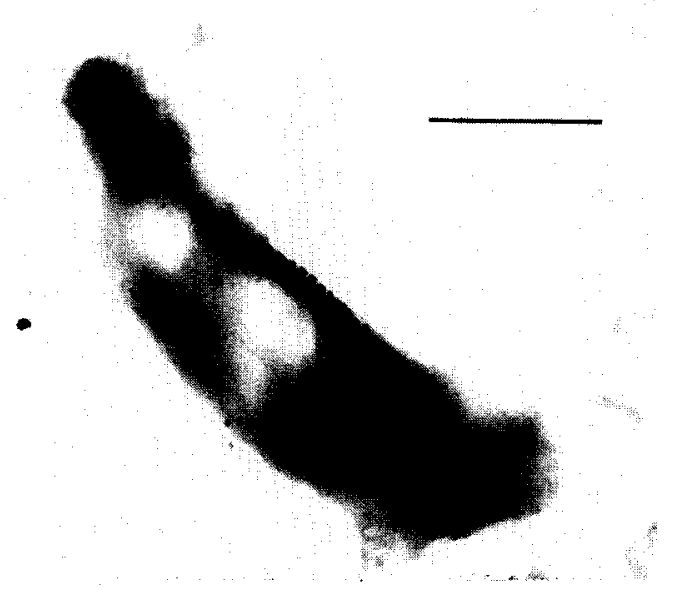

Fig. 7. Transmission electron micrograph of an uncultured magnetotactic rod (MR). Bar $=1 \mu \mathrm{m}$.

The values of $H_{\mathrm{c}}$ were $300 \mathrm{G}$ and $320 \mathrm{G}$, respectively.

\section{2. $M R$ and $M M P$}

An electron micrograph of a MR cell is shown in Fig. 7. The cell contains two adjacent chains of
$\mathrm{Fe}_{3} \mathrm{~S}_{4}$ magnetosomes. An electron micrograph of a MMP is shown in Fig. 8. The organism consists of multiple cells, each of which contains single or double magnetosome chains. The magnetosome minerals are $\mathrm{Fe}_{3} \mathrm{~S}_{4}$ and $\mathrm{FeS}_{2}$, but the arrangement of the minerals in the chains is not known. Fig. 8 shows the MMP with the individual cells disaggegated on the grid. There is evidence from electron microscopy (B.R. Heywood, S. Mann, and R.B. Frankel, unpublished) that the magnetosome chains in cells of the intact organism have a common orientation.

Pulsed field remanence plots for six MR cells and five MMP cells are shown in Figs. 9 and 10, respectively. The average values of $H_{\mathrm{c}}$ were $320 \pm 20 \mathrm{G}$ and $200 \pm 3 \mathrm{G}$, respectively. Unlike the MM cells, the MR and MMP hysteresis loops were not square, indicating that individual MR and MMP cells can be demagnetized.

\section{Discussion}

The high squareness of the pulsed remanence data obtained for MM is consistent with the single chain

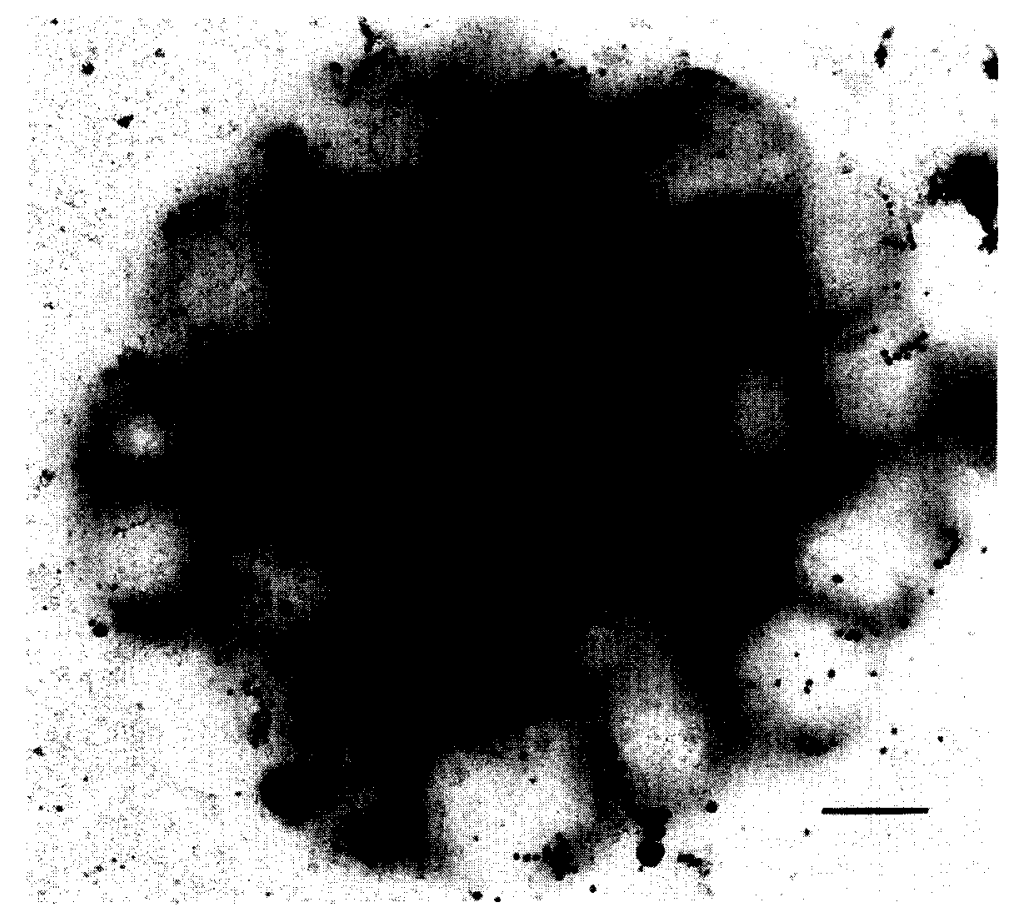

Fig. 8. Transmission electron micrograph of an uncultured, many-celled, magnetotactic prokaryote (MMP). Bar $=1 \mu \mathrm{m}$. 


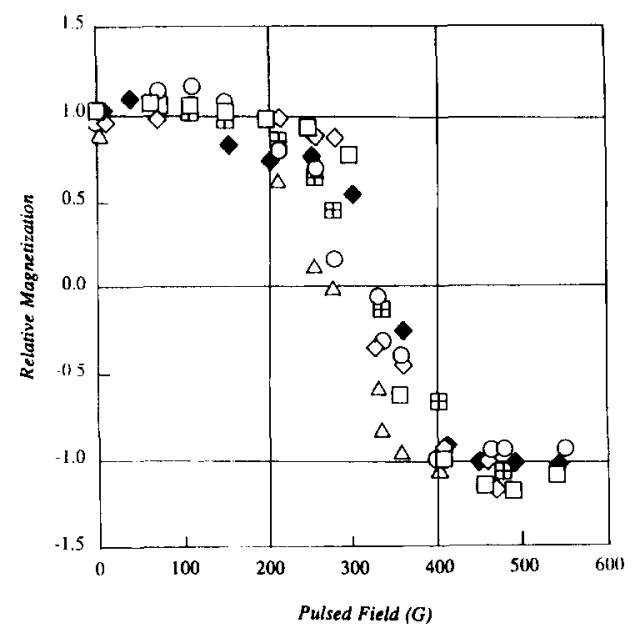

Fig. 9. Pulsed magnetic field remanence plots for a six magnetotactic rods (MR)

of single-domain magnetite particles in this organism. This structure is reminiscent of the 'chain of spheres' model for elongated single domain particles [23]. Magnetic interactions between the particles and the perfect crystallographic alignment of the crystalline easy axis along the chain direction combine to make the chain axis the easy axis of magnetization, and magnetization reversal occurs by a fanning or vortex propagation mechanism [23-25]. Because of gaps between the particles in the chain, the coercive force is less than the coercive force expected for a

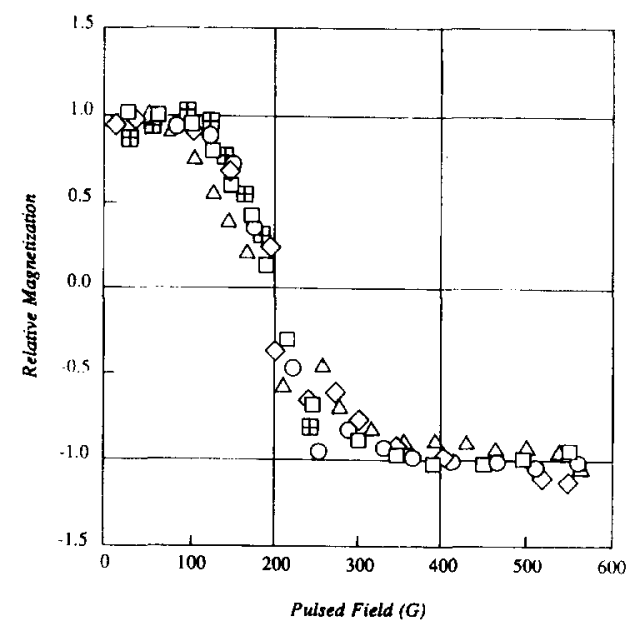

Fig. 10. Pulsed magnetic field remanence plots for five many-celled, magnetotactic prokaryotes (MMP). solid rod or cylinder [19]. For example, the classical nonsymmetric fanning mode of Jacobs and Bean [23] predicts $H_{\mathrm{c}}=439 \mathrm{G}$ for a chain of 12 magnetite particles or greater, whereas the observed $H_{\mathrm{c}}$ for the MM sample is approximately $300 \mathrm{G}$. The fanningtype reversal modes are also consistent with the observed distribution in the coercive force in the population, either through a distribution in gap sizes, chain lengths, or both. Theoretical models for fanning-like magnetization reversals in a linear chain of particles predict that chain coercivity approaches a saturation value for $n>12[23,25,26]$. We interpret the sharp cutoff in the coercivity distribution observed for the cells in terms of this saturation effect. However, since it was not possible to observe the chain length in a cell with light microscopy, it was not possible to correlate the coercive force of a given cell with chain length.

The relatively small increase, from 300 to $320 \mathrm{G}$, in the coercive force with change of angle from 0 to $45^{\circ}$ between the bacterial dipole and the pulse field is also consistent with a non-coherent reversal mode, such as the fanning mode in the chain of spheres model [23].

The sudden small decrease in moment followed by complete remagnetization at slightly higher pulse fields, observed in some MM cells, suggests that an intermediate remanent state can occasionally be obtained. The reason for this is unclear, but is probably related to departures from an ideal, linear chain structure. One type of departure is the presence of a few immature magnetosomes at the ends of chains [27]. Immature magnetosomes are smaller-sized particles, approaching the superparamagnetic-single domain transition volume, formed during initial crystal growth. The moments of these particles may switch at a lower critical field than the rest of the chain due to their smaller volumes and terminal positions. The variable occurrence of these smaller magnetosomes in $\mathrm{MM}$ cells would explain both the small magnitude of the moment decrease and why the intermediate micromagnetic state is observed in only some cells.

The pulsed hysteresis measurements on MR and MMP cells suggest that organisms with two or more adjacent chains can be effectively demagnetized. For this type of geometrical arrangement of magnetosomes, either the moments of the two strands are antiparallel ('inter-chain'), or individual magneto- 
somes along the same chain can be antiparallel ('intra-chain'). It is noteworthy that the chains with greigite only (MR) have higher coercivity than chains with greigite and pyrite (MMP), even though the greigite magnetosomes have similar size distributions in both organisms. This observation can be rationalized if we hypothesize that pyrite particles are co-organized in the chains of the MMP. Then it is likely that MR cells reverse their magnetization by inter-chain remagnetization only, whereas the MMP cells reverse their magnetization by intra-chain remagnetization. Presumably, the non-magnetic pyrite particles in the MMP chains reduce the strong positive interactions between the greigite particles, making an intra-chain reversing mode more probable with a concomitant decrease in the energy barrier for remagnetization. These observations are in agreement with recent micromagnetic modeling of switching field behavior in interacting chains of cubic and spherical particles $[24,25]$.

\section{Acknowledgements}

We thank J. Hilsinger for help in the construction of the pulsed magnetic field apparatus. IP was supported by a grant from the University of Groningen. DAB and RBF were supported by the U.S. Office of Naval Research. BMM and DAB were supported by the National Science Foundation. Support for the Institute for Rock Magnetism was provided by grants from the Keck Foundation and the National Science Foundation. This is contribution 9409 of the Institute for Rock Magnetism.

\section{References}

[1] R.P. Blakemore, N.A. Blakemore, D.A. Bazylinski and T.T. Moench, in: Bergy's Manual of Systematic Bacteriology, Vol. 3, J.T. Staley, E.P. Bryant, N. Pfennig and J.G. Holt, eds. (Williams and Wilkins, Baltimore, MD, 1989) p. 1882.

[2] D.A. Bazylinski, A.J. Garratt-Reed and R.B. Frankel, Microsc. Res. Tech. 27 (1994) 389.
[3] R.B. Frankel, R.P. Blakemore and R.S. Wolfe, Science 203 (1979) 1355.

[4] R.F. Butler and S.K. Banerjee, J. Geophys. Res. 80 (1975) 4049 .

[5] S. Mann, N.H.C. Sparks, R.B. Frankel, D.A. Bazylinski and H.J. Jannasch, Nature 343 (1990) 258.

[6] B.R. Heywood, D.A. Bazylinski, A.J. Garratt-Reed, S. Mann and R.B. Franke], Naturwissenschaften 77 (1990) 536.

[7] F.G. Rogers, R.P. Blakemore, N.A. Blakemore, R.B. Frankel, D.A. Bazylinski, D. Maratea and C. Rogers, Arch. Microbiol. 154 (1990) 18.

[8] D.A. Bazylinski, B.R. Heywood, S. Mann and R.B. Frankel, Nature 366 (1993) 218.

[9] R.B. Frankel, Ann. Rev. Biophys. Bioeng. 13 (1984) 85.

[10] A.J. Kalmijn and R.P. Blakemore, in: Animal Migration, Navigation and Homing, eds. K. Schmidt-Koenig and W.T. Keeton (Springer-Verlag, Berlin, 1978) p. 354.

[11] M. Mizota and Y. Maeda, Annu. Rep. Res. Reactor Inst. Kyoto Univ. 16 (1983) 144.

[12] R.P. Blakemore, R.B. Frankel and A.J. Kalmijn, Nature 286 (1980) 384.

[13] J.E. Knowles, IEEE Trans. Magn. 14 (1978) 858.

[14] R.P. Blakemore, D. Maratea and R.S. Wolfe, J. Bacteriol. 140 (1979) 720.

[15] R.S. Wolfe, R.K. Thauer and N. Pfennig, FEMS Microbiol. Ecol. 4 (1987) 31.

[16] C.P. Bean, Biophys. J. 54 (1989) 258a.

[17] H.C. Berg, Random Walks in Biology (Princeton University Press, Princeton, NJ, 1983) p. 83.

[18] N. Petersen, D.G. Weiss and H. Vali, in: Geomagnetism and Palaeomagnetism, eds. F.J. Lowes, D.W. Collison, J.H. Parry, S.K. Runcorn, D.C. Tozer and A. Soward (Kluwer Academic Publishers, Amsterdam, 1989) p. 231.

[19] B.M. Moskowitz, R.B. Frankel, P.J. Flanders, R.P. Blakemore and B.B. Schwartz, J. Magn. Magn. Mater. 73 (1988) 273-288.

[20] R.W. Chantrell and K. O'Grady, in: Magnetic Properties of Fine Particles, eds. J.L. Dorman and D. Fiorani (North-Holland, Amsterdam, 1992) p. 103.

[21] R. Proksch and B.M. Moskowitz, J. Appl. Phys. 75 (1994) 5894.

[22] B.M. Moskowitz, R.B. Frankel and D.A. Bazylinski, Earth Planet. Sci. Letters 120 (1993) 283.

[23] I.S. Jacobs and C.P. Bean, Phys. Rev. 100 (1955) 1060.

[24] Y. Uesaka, Y. Nakatani and N. Hayashi, J. Magn. Magn. Mater. 123 (1993) 209.

[25] P.V. Hendriksen, G. Christiansen and S. Morup, J. Magn. Magn. Mater. 132 (1994) 207.

[26] P.C. Kuo, J. Appl. Phys. 64 (1988) 5071.

[27] S. Mann, R.B. Frankel and R.P. Blakemore, Nature 310 (1984) 405 